\title{
EXPERIMENTAL VALIDATION OF A NONLINEAR MPC STRATEGY FOR A WAVE ENERGY CONVERTER PROTOTYPE
}

\author{
Hoai-Nam Nguyen* \\ Guillaume Sabiron \\ Paolino Tona \\ IFP Energies nouvelles \\ Rond-point de l'échangeur de Solaize \\ BP 369360 Solaize, France \\ Email: name.surname@ifpen.fr
}

\author{
Morten Mejlhede Kramer \\ Aalborg University \\ Department of Civil Engineering \\ Wave Energy Research Group \\ Sofiendalsvej 11 \\ DK-9200 Aalborg, Denmark \\ Email: mmk@civil.aau.dk
}

\author{
Enrique Vidal Sanchez \\ Wave Star A/S \\ Park All 350E \\ DK-2605 Brndby, Denmark \\ Email: evs@wavestarenergy.com
}

\begin{abstract}
One of the major limitations to the development of advanced wave energy converters (WECS) control strategies are the associated computational costs. For instance, model predictive control $(M P C)$ strategies have the potential to obtain almost optimal performance, provided that the imperfect power conversion in the power take-off (PTO) system is correctly taken into account in the optimization criterion and that the incoming wave force can be estimated and forecast. However, demanding computational requirements as well as the unresolved issue of wave force estimation have so far prevented real-time implementation and validation of such MPC strategies. In this paper, we present the successful experimental results obtained on a scaled-down prototype of the well-known Wavestar machine. Performance comparisons are provided for nonlinear MPC versus a reference PI controller.
\end{abstract}

\section{INTRODUCTION}

A wave energy converter (WEC) is a device used to produce electricity, or other forms of usable energy, from wave motion. The main challenge faced by the developers of wave energy technologies is the reduction of the levelized cost of energy (LCOE) to a competitive level. A key driver to achieve such a goal is the improvement of "wave-to-wire" efficiency, which, especially for point absorbers of the heaving-buoy type, depends on:

- their architecture (geometry, mechanics),

- the efficiency of the power take-off (PTO) system

- the performance of the PTO control system [1].

${ }^{*}$ Address all correspondence to this author.
In order to deal with the naturally narrow-banded frequency response of such dynamic systems, and the continuously changing sea state, flexible PTOs capable of both harvesting and drawing power from the grid (respectively in generator and motor modes) are promising actuator candidates. A flexible PTO along with reactive control allows the absorber to be more often in phase with the incoming waves. It can achieve that, by investing some energy (drawn from the grid) to eventually get a larger energy payback than it would be possible to obtain by just braking the absorber via the PTO force. Indeed, many studies have shown that one of the key aspects for maximizing the energy yield of a WEC is the way of controlling the device. The ProportionalIntegral (PI) velocity feedback controller is the current state of the art for WECs as far as practical implementation is concerned. The integral action is a position feedback implementing a simple form of reactive control, while the proportional velocity feedback provides the basic linear damping which is found in most WEC control system. This strategy is very robust and simple to implement, since it uses only position and velocity measurements to compute the control action. The PI control law shows a reasonable energy conversion rate, but is still far below the theoretical optimum discussed in [2]. Moreover, additional performance loss is to be expected because the sea state changes and the feedback coefficients must be modified online to take into account this variation.

Of course, alternatives to PI control do exist. Latching control has been proposed for WECs equipped with a position locking mechanism [3-5]. The basic idea is to lock the point absorber when its velocity is zero, and wait for the most favorable moment to release it again. In this way, the velocity of the point absorber can be brought in phase with the wave excitation force, and the 
system is in resonance. When the point absorber is unlatched, energy is recovered via proportional velocity feedback. In [6], by combining latching control and short term wave prediction, it was shown that the performance can be improved up to $20 \%$ compared to the classical PI control law.

Another solution, that has been drawing a lot of attention in the last decade, is Model Predictive Control (MPC) [7-9]. In MPC, a system model is used to predict the future response of a plant. At each control interval, the algorithm attempts to optimize future plant behavior by computing a sequence of future control actions. The first input in the optimal control sequence is then applied to the plant, and the entire calculation is repeated at subsequent control intervals. The main advantage of MPC is that it is capable of energy conversion rates close to the theoretical optimum. In addition, state and input constraints can be considered in a natural way. However, MPC is computationally demanding, since a wave prediction procedure and an iterative optimization have to be carried out for each control interval. Up to now, only linear MPC algorithms using precomputed predictions have been implemented in real-time on WECs [10].

In [11], another approach to improve performance is proposed, the so called simple and effective real-time control. Based on the assumption of the availability of wave excitation force, the basic idea is to calculate a reference optimal velocity. Then a control law is designed to make the float velocity follow the reference one. By simulation, it was reported that performance can be improved up to $25 \%$ compared to the PI control law.

To achieve optimal energy conversion, the three aforementioned strategies rely on the assumption that the instantaneous wave excitation force is available. However, this is not the case in practical WEC implementations, since this force cannot be measured directly when the WECs are in operation. Therefore, the force has to be estimated using other quantities, e.g. the modeled radiation force, or via an observer.

In [12], using a combination of a bank of independent harmonic oscillators and a Luenberger observer, an estimation algorithm is proposed. The strategy was tested on a real WEC system, but the experimental results showed a significant phase lag in the estimated signal compared to the measured signal.

In [13], by considering the wave excitation torque as a timevarying sinusoid, an extended Kalman filter approach is presented. However, no experimental results are reported. In addition, it is obvious that the approach may only be effective for very narrow-banded wave forces.

In this paper, the implementation of a complete nonlinear MPC control control system on a point-absorber WEC is presented and discussed. The emphasis is placed on experimental results collected in the wave basin of Aalborg University, in June 2015, on a small-scale wave energy converter prototype, manufactured by the Wavestar company. All the implementation aspects, from computational complexity to accuracy, are investigated. First, the main building blocks of the MPC control system are briefly introduced including:

- an online wave excitation torque estimation algorithm using only available measurements (float position and velocity, PTO force) yielding estimations with no significant time delay (contrary to [12]),

- an accurate and self-adaptive short-term wave force prediction algorithm,

- a real-time compatible nonlinear MPC algorithm taking into account PTO conversion efficiency.

Then, experimental results are presented and analyzed. MPC performance is compared against the performance of a reference PI control scheme on a time-varying sea state in terms of harvested power and control input signal.

The paper is organized as follows. Section 1 introduces the model of the wave energy converter considered in this study. In section 2 the different building blocks listed above are briefly described. Experimental setup and results are presented in section 3. Finally, section 4 summarizes the overall conclusions and proposes further stages of investigation.

\section{WEC modeling}

The Wavestar device extracts energy from the pivoting motion of buoys connected to a platform. In the following we consider a single-float configuration. It is assumed that the float motion can be described by a simple model derived from linear wave theory:

$$
J \ddot{\theta}(t)=M_{e x}(t)-M_{P T O}(t)-M_{h y d}(t)-M_{r a d}(t)
$$

where $J$ is the total moment of inertia of arm and float, $\theta$ is the float angular position, $M_{e x}$ is the wave excitation moment of force, including diffraction effects, $M_{P T O}$ is the moment of force applied by the PTO, $M_{h y d}$ is the hydrostatic restoring moment of force and $M_{r a d}$ the radiation moment of force. In the following we will use the term "torque" for moment of force.

For small $\theta(t), M_{h d}(t)=-K_{r e s} \theta(t)$, where $K_{r e s}$ is the hydrostatic stiffness coefficient.

The radiation term is written as by

$$
M_{\text {rad }}(t)=-J_{\infty} \ddot{\theta}(t)-M_{r}(t)
$$

where $J_{\infty}$ is the added inertia at infinitely high frequency, and

$$
M_{r}(t)=\int_{0}^{t} h(t-\tau) \dot{\theta}(\tau) d \tau
$$

where $h(t)$ is the impulse response function of the radiation moment, obtained by boundary element methods. 
Introducing a linear time invariant realization for Eqn. 3, via the transfer function $W_{r}(s)$

$$
M_{r}(s)=W_{r}(s) \dot{\theta}(s)
$$

the system model can be written in the Laplace domain as

$$
\left(\left(J+J_{\infty}\right) s+\frac{K_{r e s}}{s}+W_{r}(s)\right) \dot{\theta}(s)=M_{e x}(s)-M_{u}(s)
$$

Introducing $J_{e q}=J+J_{\infty}$, the resulting system model can be represented in block-diagram form as shown in Fig. 1.

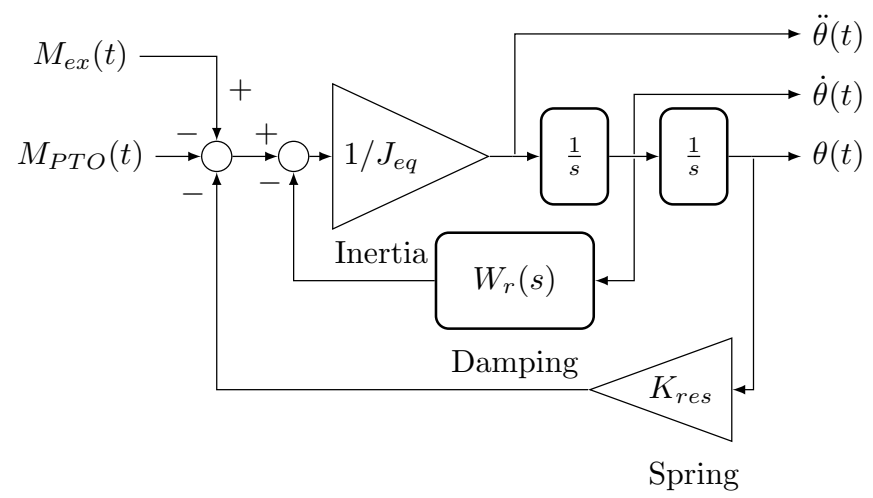

FIGURE 1: SYSTEM MODEL IN BLOCK-DIAGRAM FORM

\section{NON LINEAR MODEL PREDICTIVE APPROACH 2.1 Wave Torque Estimation And Prediction}

If the wave excitation torque is known and it is possible to predict its future evolution over a short time horizon, then the whole future dynamic behavior of the WEC can be predicted assuming that its motion can be described by Eqn. (1).

Unfortunately, wave excitation torque $M_{\text {ext }}(t)$ is not directly available online during normal WEC operation (it is usually measured offline using a dedicated experiment where the float is blocked and a force/torque sensor measures the effect of the wave on the WEC). Thus, an algorithm is needed which performs the estimation of wave excitation torque from standard WEC measurements.

The estimation method developed by the authors to tackle this issue considers the variation of the excitation moment as a random signal. Based on a Kalman filter and a random walk model for the wave excitation torque, the estimation problem is transformed into a classical state estimation problem.

The main features of this estimation method are:
1. estimated wave torque values do not show any significant lag compared to "true" values, which is not the case for the approach in [12];

2. in contrast to [12], no (implicit) unrealistic assumption about the time-invariant nature of the sea state is made, hence any operating condition can be efficiently dealt with;

3. state and control constraints can be considered;

4. in addition to the wave excitation torque, the estimates of the state of the system can also be provided, to be used for advanced control algorithms such as MPC.

The above-mentioned algorithm makes an estimated wave force (moment) value available at each instant $k$. The present and past values of (estimated) wave torque can be used to form a time series, whose future evolution can be predicted via an autoregressive model:

$M_{e x}(k)=a_{1} M_{e x}(k-1)+a_{2} M_{e x}(k-2)+\ldots+a_{p} M_{e x}(k-p)+w(k)$

where $w(k)$ is a stochastic uncertainty, a random noise with zero mean. As the sea state is not stationary, we cannot expect good prediction results by simply estimating the AR model parameters via the minimization of the one-step ahead prediction error. One possible strategy (proposed by F. Fusco and J. Ringwood of the University of Maynooth in [14]) consists in finding the AR parameters which minimize the prediction error over multiple steps:

$$
\min _{a_{1} \ldots a_{p}} \sum_{l=p+h+1}^{k} \sum_{j=1}^{h}\left(M_{e x}(l)-\hat{M}_{e x}(l \mid l-j)\right)^{2}
$$

using (long) data batches, and recomputing them at regular times (or each time the sea state changes). This technique, also known as long range predictive identification ([15]) requires solving a computationally-demanding nonlinear least square optimization problem.

The authors have developed an alternative method, also based on AR model estimation, to overcome the practical drawbacks of the aforementioned strategy. An adaptative bank of Kalman filters is used for this method, which:

- does not need explicit low-pass filtering of data;

- has a much lower computational complexity;

- updates unknown parameters as soon as new information is available, eliminating the need for a supervisory layer triggering the recomputation of the AR model parameters;

- works well for a (wide) range of (fixed) sampling periods.

\subsection{Model Predictive Control}

The main role of the PTO control system is to take off as much energy as possible from the waves to the network, for a broad range of sea states. 


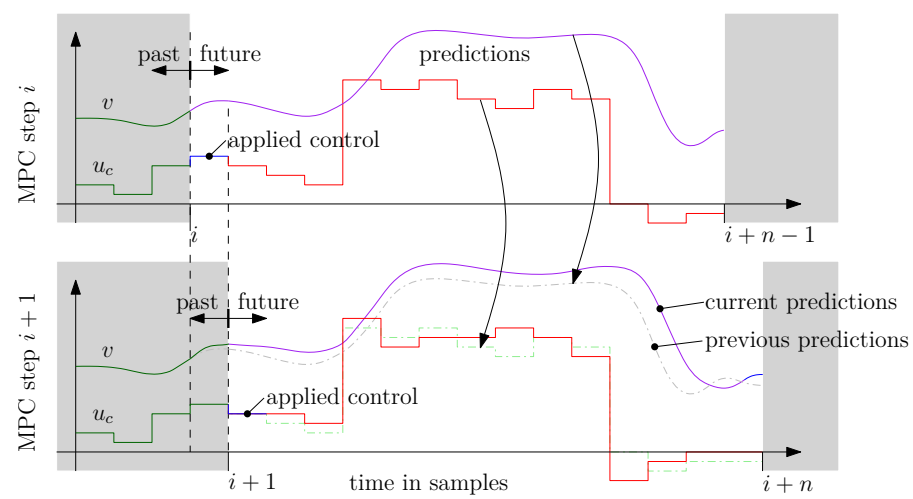

FIGURE 2: THE PRINCIPLE OF SLIDING HORIZON MPC.

PI velocity feedback control (also referred to as $B K$-control in [16]) is the control strategy currently favored by Wavestar:

$$
u_{c}(t)=M_{P T O}(t)=-k_{p} \int_{t_{0}}^{t} \omega(\tau) \mathrm{d} \tau-k_{v} \omega(t)
$$

where $u_{c}$ is the control input signal corresponding to the PTO force setpoint. To achieve good performance, parameters $k_{p}$ and $k_{v}$ must be tuned for the current sea state using a model-based approach or by trial and error. This strategy is simple and robust, even though it is suboptimal and requires a switching of control parameters triggered by a supervisory layer.

Among the implementable control strategies, MPC has instead the potential to lead to almost optimal performance, because of its ability to deal with many design objectives and performance criteria and to handle constraints on states and control inputs.

MPC is a receding horizon strategy which consists of the following steps:

1. At current sample $i$, measure (or estimate) the system state and use it as an initial value to calculate an optimal control over a limited horizon with $n$ samples starting at sample $i$. This yields a time series of $n$ optimal control values $u_{c, i}$.

2. Apply the first value of $u_{c, i}$ (corresponding to the control for the current sample) as a command for the system and hold it for the duration of the sample period.

3. At the next sample $i+1$, measure the system state and use it again as an initial value to calculate an optimal control over a horizon with $n$ samples starting at $i+1$. Initialize the vector of decision variables using the results from the previous step. This yields $u_{c, i+1}$.

4. Apply the first value of $u_{c, i+1}$.

This principle is depicted in Figure 2.

MPC is actually implementable in real-word applications as an online control law if a short-term wave prediction is available, provided that its computational time is short enough compared with the control sampling time (which is in turn determined by the system bandwidth).

Examples of MPC strategies, based on linear or nonlinear models, convex or non-convex criteria, can be found in [17-21]. Most strategies are formulated so as to maximize the net produced energy over a given horizon, which corresponds (for large enough horizons) to the ultimate goal any WEC control system should strive for. However, a critical factor is often overlooked in the design and validation of MPC (as well as of most other WEC control strategies): the imperfect power conversion in the PTO. Even though this practice is seldom followed even in the most recent literature, conversion efficiency should be always taken into account in simulation and during the experiments. In simulation, this is necessary in order to obtain realistic results and a fair comparison among controllers, as shown in [16] for PI velocity feedback control and in [22] for latching control.

In [23], IFPEN has presented a nonlinear MPC strategy for a generic heaving-buoy converter (the first one proposed so far, to the extent of the authors' knowledge) which takes directly into account the imperfect power conversion in the PTO. This is achieved by introducing a (nonlinear) conversion efficiency, function of the instantaneous power, in the optimization criterion. For the pivoting-buoy setup considered here, the control objective corresponds to the minimization of the mean electrical power:

$$
\bar{P}_{e}=-\frac{1}{T} \int_{t=0}^{T} \eta P_{e} \mathrm{~d} t, \quad P_{e}=\eta P_{a}=\eta u \omega .
$$

which is given a negative sign since is taken from the system. $\bar{P}_{e}$ is thus calculated as the normed integral over time $T$ of the instantaneous electric power $P_{e}$, which is in turn given by the product of PTO torque $u$ and float angular velocity $\omega$ (i.e., the ideal hydrodynamical power $P_{a}$ ) multiplied by PTO efficiency $\eta$.

The efficiency $\eta$ is taken as a function of $P_{a}=u \omega$ :

$$
\eta(u \omega)=\left\{\begin{array}{l}
\eta_{0} \text { if } u \omega \geq 0 \\
\frac{1}{\eta_{0}} \text { if } u \omega<0
\end{array} \quad, 0<\eta_{0} \leq 1\right.
$$

The coefficient $\eta_{0}$ corresponds to the product of the average efficiencies of each PTO stage (actuator, hydraulic transmission if any, generator/motor, inverters). More generic functions of $P_{a}$ could also be used, though this would likely complexify the optimization process.

With the above formulation, MPC "knows" that, when $\eta_{0}<$ 1 , the system will produce $P_{e}<P_{a}$ when generating and consume $P_{e}>P_{a}$ when motoring.

The control model used for MPC is derived from Eqn. 5, converted in state-space form and time-discretized. This allows 
to express the power to be minimized as a function of $u_{c}$, the vector of control values applied over the next $N_{p}$ steps $\left(N_{p}\right.$ is called the prediction horizon). After some manipulations (detailed in [23]), the discretized mean power to be optimized can be put in the convenient quadratic form

$$
\mathscr{P}_{e}\left(u_{c}\right)=1 / 2 u_{c}^{T} \boldsymbol{H}\left(u_{c}\right) u_{c}+\boldsymbol{f}\left(u_{c}\right)^{T} u_{c}+c_{0}\left(u_{c}\right)
$$

where the matrix $\boldsymbol{H}$ and the vectors $\boldsymbol{f}$ and $\boldsymbol{c}_{\mathbf{0}}$, are obtained from the matrices of the control model state-space representation.

Unfortunately, the objective function introduced above is non-convex, which makes the optimization problem difficult and costly to solve. Indeed, the strategy developed in [23] cannot be applied "as is" to the small-scale prototype we consider here, as its dynamics requires a control sampling period not longer than $50-100 \mathrm{~ms}$, which is barely enough to compute the optimal solution, in simulation, on a standard modern laptop (it could still prove real-time compatible on the full-scale system, though, since the control sampling period would be much longer).

In order to have a nonlinear MPC running on the small-scale prototype at AAU, several variants of this strategy were developed and tested in simulation. Two methods were finally selected, whose computational costs are significantly reduced with respect to the original strategy, thanks in particular to the convexification of the objective function. One of these methods (the one with the best performance in simulation) has been chosen for experimental testing.

\section{FIELD TESTS RESULTS}

\subsection{Test Facility and Setup}

A laboratory prototype of a point absorber WEC on a $1: 20$ scale with respect to the well-known Wavestar machine installed near Hanstholm, in Denmark, was used to test IFPEN control system, see Figure 3 . The prototype consists of a float attached to an arm, which in turn is attached to a power take-off system (PTO). The laboratory model is equipped with position and acceleration sensors. The velocity of the float is estimated from the position and the acceleration via a linear Kalman filter.

The Aalborg University wave basin is equipped with 16 wave gauges and is $8.5 \mathrm{~m}$ wide and $15.7 \mathrm{~m}$ long. The wave paddles are driven by a total of 15 hydraulic pistons moving in the horizontal direction. The waves used for the tests were generated by the wave maker based on a Pierson-Moskowitz spectrum.

\subsection{Control Design and Implementation}

The state-space model for MPC design has been computed from the parameters given in Table 1.

PTO dynamics was initially neglected, as it was considered much faster than WEC dynamics. Notice that, while the strategy

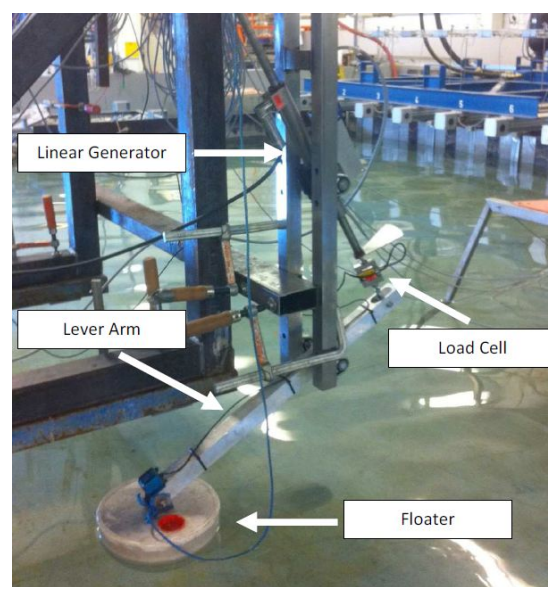

FIGURE 3: LABORATORY WEC LAYOUT.

\begin{tabular}{|l|l|l|}
\hline \multicolumn{3}{|c|}{ Hydrodynamic model parameters } \\
\hline Inertia of arm and float & $J$ & $1.04 \mathrm{~kg} \mathrm{~m}^{2}$ \\
Hydrostatic stiffness coefficient & $K$ & $93 \mathrm{~N} \mathrm{~m} \mathrm{rad}^{-1}$ \\
Added inertia & $J_{\infty}$ & $0.40 \mathrm{~kg} \mathrm{~m}^{2}$ \\
\hline \multicolumn{2}{|c|}{ Radiation moment impulse response realization } \\
\hline \multicolumn{2}{|c|}{$W_{r}(s)=\frac{0.0312 s^{3}+34.79 s^{2}+3770 s+760.8}{s^{3}+124.2 s^{2}+1498 s+1.064004}$} \\
\hline
\end{tabular}

TABLE 1: PARAMETER VALUES FOR THE LABORATORY WAVESTAR PROTOTYPE.

in [23] can handle PTO dynamics at design level, by including it in the control model, the MPC formulation under test can only do that via the optimization of some internal weightings to be performed off-line on a simulation model including both WEC and PTO dynamics.

The other relevant MPC parameters are:

- Control sampling period $T_{M P C}=50 \mathrm{~ms}$;

- Prediction horizon $N_{p}=25$;

- Mean PTO efficiency $\eta_{0}=0.7$;

- Control limits $u_{\min }=-6.5 \mathrm{Nm}, u_{\max }=6.5 \mathrm{Nm}$;

The control limits, chosen to match those of the full-scale PTO, are enforced by the software. The actuator of the scaled-down setup could actually handle more than $6.5 \mathrm{Nm}$. Position limits are not considered as they are not hit during normal tests.

The Simulink diagram describing the whole MPC control system is represented in Fig. 4. One can recognize the three main building blocks with different sampling rates: wave and state estimator running at $200 \mathrm{~Hz}$, the wave prediction algorithm and finally the MPC strategy, both running at $20 \mathrm{~Hz}$.

The first experimental results showed that, with the current tuning of the force servo, PTO dynamics was actually too slow 


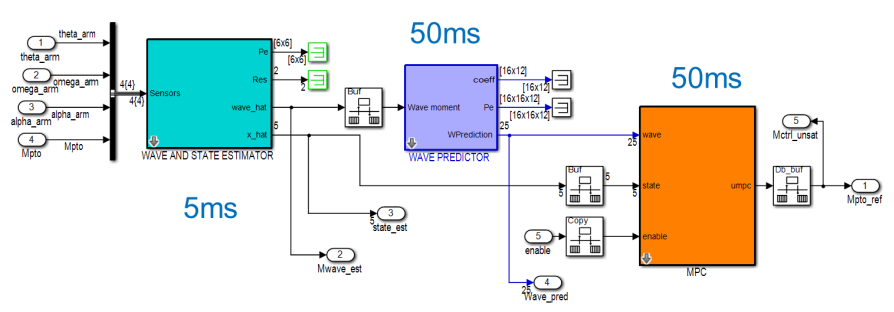

FIGURE 4: MPC COMPLETE SYSTEM AS IMPLEMENTED IN AAU WAVE BASIN.

to be neglected. To cope with this, the initial MPC calibration had to be changed. As explained before, an optimization of internal MPC weightings was run in simulation, considering both the system dynamics and the PTO dynamics, for all the expected sea states. Due to the short time window available for the computationally-intensive optimization procedure, the implemented parameters were suboptimal (as the procedure had not terminated).

\subsection{Methodology}

The experiments were performed for 5 available sea states representative of real-life conditions. Figure 5 presents the power spectral density of the 4 different basic sea states. The last one denoted S23 correspond to a time-varying sea state switching linearly from S2 to S3. It is important to note that the most interesting sea state from an energy point of view are $\mathrm{S} 2$ and $\mathrm{S} 3$ since they correspond to the most frequent sea states and contain much more energy than S1. S4 might be seen as a critical sea state which might require to lift up the float out of the water. All available sea states are composed of irregular waves.

For each sea state we ran a series of tests starting with reference PI controllers tuned offline by Wavestar. Each PI controller, intended to provide a performance benchmark with respect to the MPC algorithm, was optimized for a given sea state. Then, we tested the MPC strategy, first with initial calibration, then with the new set of parameters obtained by (partial) optimization of harvested power including PTO dynamics in the plant model.

Notice that, in real-life conditions, the sea state would

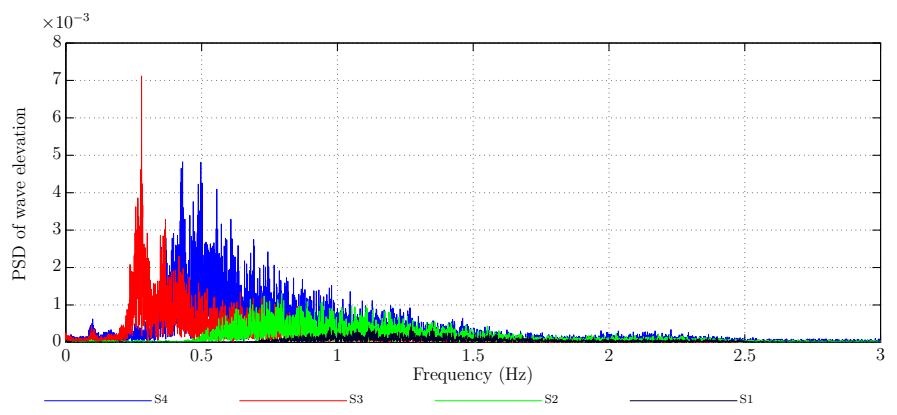

FIGURE 5: WAVE ELEVATION PSD AT FLOAT LOCATION.

\begin{tabular}{c|c|l} 
Control & Name & Comment \\
\hline \hline Reference PI & Classic Si & PI optimized offline for Si \\
\hline \multirow{2}{*}{ MPC } & MPC Si & $\begin{array}{l}\text { MPC with weigthings } \\
\text { optimized for S1-S4 and } \\
\text { no PTO dynamics }\end{array}$ \\
\cline { 2 - 3 } & MPC Si PTO & $\begin{array}{l}\text { MPC with weigthings } \\
\text { optimized for S2-S4 and } \\
\text { PTO dynamics }\end{array}$ \\
\hline
\end{tabular}

TABLE 2: CONTROL STRATEGIES TESTED IN THE AAU WAVE BASIN.

change continuously which would require an intervention, be it human or automated, to switch from one set of PI gains to another. To deal with time-varying sea states, instead, the MPC approach does not need any intervention from a supervisory layer nor any manual retuning, since sea state parameters are estimated online and a wave prediction is performed for MPC. Thus, it is to be expected that the gains obtained in terms of harvested power be much higher with the wave S23, as the fixed-gain PI control strategy used as a benchmark is unable to smoothly adapt to the current sea state. Of course, PI control performance can be improved with a supervisory layer, which could identify the current sea state and select the most suited precomputed gains for it. But this basic strategy, similar to gain scheduling techniques, cannot bridge the gap with MPC as it will certainly suffer from time delays during sea state changes (some time might be needed to ensure that the sea state has changed from A to B).

\subsection{Results}

To help the reader with the figures shown in the following, Table 2 reports the nomenclature adopted for the experimental testing of the various control strategies, including four PI controllers (each calibrated for a specific sea state) and two MPC controllers with a different calibration.

Table 3 presents the MPC results for all the performed experiments in terms of percent of harvested power compared to the reference PI controller. One can see that for all the sea states, the gain is positive ranging from $7 \%$ to $81.5 \%$. Table 4 gives more details on the experimental results of each tests.

\begin{tabular}{l|ccccc} 
Wave & S1 & S2 & S3 & S4 & S23 \\
\hline \hline MPC & 41.4 & 7.6 & - & - & - \\
MPC PTO & - & 15.7 & 20.9 & 8.7 & 81.5
\end{tabular}

TABLE 3: GAIN (IN \% OF $P_{E}$ ) FOR MPC VS. REFERENCE PI. 


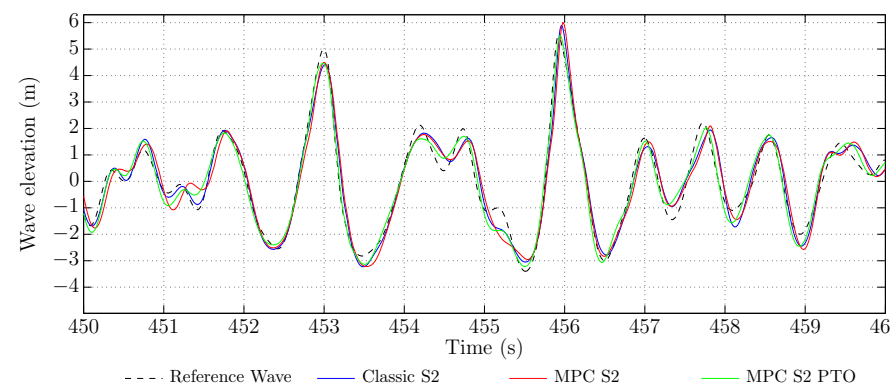

FIGURE 6: WAVE ELEVATION FOR S2, UPSTREAM OF THE FLOAT

Several observations can be made:

- The MPC algorithm has to take into account PTO dynamics. One can see that in the MPC version which neglects PTO dynamics the gain on $\mathrm{S} 2$ is very small compared to the gain obtained on S1. This could be explained by the fact that $\mathrm{S} 1$ being quite slower than S2 the control signal delivered to the actuator did not excite the faster dynamics of the PTO. Using the version where PTO dynamics is taken into account at calibration level, the MPC results were quite good for S2 to $\mathrm{S} 23$.

- As expected, the highest improvements were obtained on S23 where the sea state was linearly changing with time.

Wave Elevation It is important to notice that even if the basin paddles are supposed to create the same wave from one experiment to the other, small variations are observed from one experiment to another. These differences can be seen on the zoomed view of the wave elevation on Figure 6. The wave elevation signals correspond to the measurement of the first wave gauge of the basin that is supposed to be the least disturbed by the refracted wave from the float. The differences between the waves are twofold: firstly, a time delay up to 0.07 seconds and, secondly, an amplitude difference (RMSE up to $0.3 \%$ ).

In order to validate the designed control strategy, one have to keep in mind that these discrepancies over the repeatability of the generated wave might induce differences on the observed performance, especially concerning the wave torque estimation part.

Wave Torque Estimation Figure 7 shows the estimated wave excitation torque for S2 and for all the tested control strategies where this algorithm was implemented (MPC, but not reference PI). The reference wave corresponds to a previously run experiment with a fixed float which allowed the PTO force sensor to measure the wave excitation force (then transformed into wave excitation torque). The wave torque estimation algorithm performed well for the 5 sea states with no noticeable time delay. It

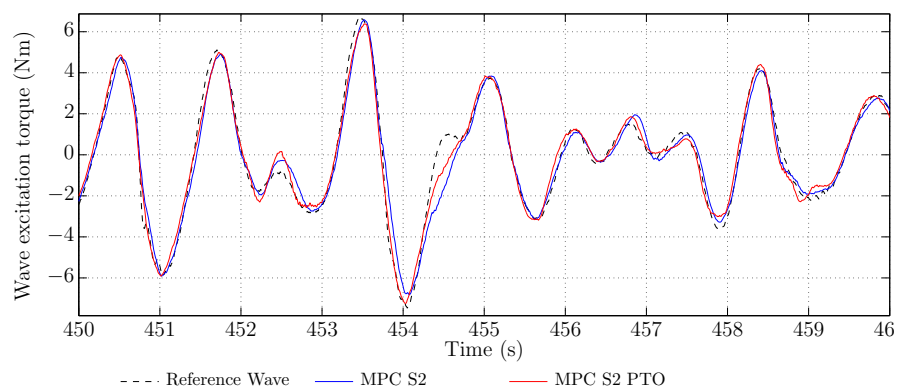

FIGURE 7: ESTIMATED WAVE EXCITATION TORQUE FOR S2.

is important to notice that the performance of this algorithm does not depend on the control strategy implemented. Small differences with respect to the reference wave can in part be explained by the discrepancies between generated waves as discussed in the previous section.

Wave Torque Prediction As explained in the previous chapter, the MPC algorithm requires a short-term prediction of the wave excitation torque. The prediction horizon duration was set to 25 samples (the prediction was computed each 50ms which gives $1.25 \mathrm{~s}$ ). Figure 8 shows the predicted wave excitation torque for S3. The blue line represents the reference wave excitation torque and the red one shows the estimated excitation torque. On Figure 8, the top figure shows the full prediction (25 steps) and the bottom figure shows only the first 4 steps of the prediction. This highlights the fact that the prediction is quite accurate during the first few steps and also that even if the error in the prediction can be large at the end of the 25 steps, this error is not propagated since the prediction vector is recomputed again at the next sampling time, and the algorithm does not use the previous predictions as an input. A thorough study could be realized to analyze the impact of prediction quality on control performance and find the most suitable trade off for the prediction horizon. On the one hand, a longer horizon allows the MPC to "see further", on the other hand, it induces larger prediction errors towards the end of the horizon. For a matter of clarity, only one prediction out of ten is depicted on the figures (a new prediction every 500ms).

The root mean square error (RMSE) can be used to assess the goodness of fit between the predicted wave force and the estimated one. As expected, the RMSE is smaller with a shorter prediction horizon. Figure 9 presents the RMSE between the estimated wave excitation torque and the predicted one. The 25 steps are considered with the blue line and only the 4 first steps are considered with the red one. Only one prediction out of ten is used for the RMSE computation. 


\begin{tabular}{l|cccccccccc} 
Wave & \multicolumn{3}{|c}{ S1 } & \multicolumn{2}{c}{ S2 } & \multicolumn{2}{c}{ S3 } & \multicolumn{2}{c}{ S4 } & \multicolumn{2}{c}{ S23 } \\
\hline \hline Power $[\mathrm{mW}]$ & $\mathrm{Pa}$ & $\mathrm{Pe}$ & $\mathrm{Pa}$ & $\mathrm{Pe}$ & $\mathrm{Pa}$ & $\mathrm{Pe}$ & $\mathrm{Pa}$ & $\mathrm{Pe}$ & $\mathrm{Pa}$ & $\mathrm{Pe}$ \\
\hline Reference PIs & 22 & 15 & 397 & 237 & 544 & 344 & 1123 & 727 & 361 & 250 \\
MPC & 32 & 21 & 420 & 255 & - & - & - & - & - & - \\
MPC PTO & - & - & 445 & 274 & 625 & 416 & 1159 & 791 & 699 & 454
\end{tabular}

TABLE 4: GAIN (IN [W] OF $P_{A}$ AND $P_{E}$ ) FOR THE EVALUATED CONTROL STRATEGIES. (-) INDICATES TESTS NOT PERFORMED DURING THE 3 DAYS TEST SESSION.

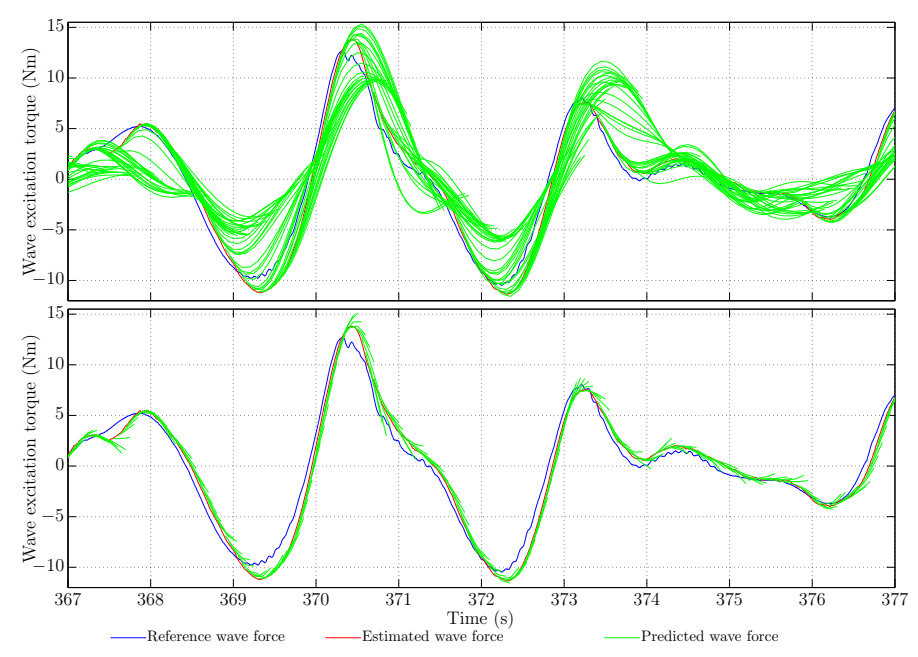

FIGURE 8: PREDICTED WAVE EXCITATION TORQUE FOR S3.

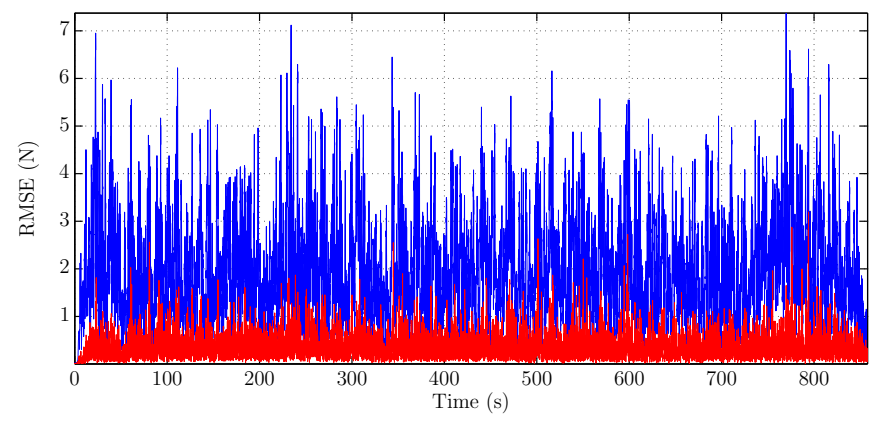

— RMSE 25 steps — RMSE 4 steps

\section{FIGURE 9: RMSE BETWEEN PREDICTED WAVE MOMENT} AND ESTIMATED ONE FOR S3.

Control Input Figure 10 shows a zoomed view of the control input signal after the physical saturation for sea state S23. Indeed, one of the advantages of the MPC is that the control saturation is taken into account which avoids undesired saturation, in a predictive way. Analyzing this signal for all sea states, several observations can be made:

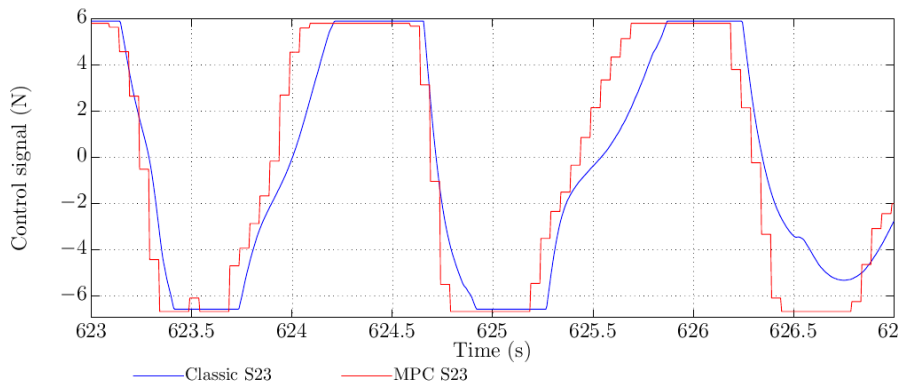

FIGURE 10: SATURATED COMPUTED CONTROL SIGNAL. SEA STATE S23.

- with S1, the control input signal are quite far from the saturation of $6.5 \mathrm{Nm}$;

- from S2 to S23, all control strategies reach the saturation;

- for stronger sea state condition (S4 for instance) MPC tends to deliver a bang-bang type control input signal.

Harvested Power Figure 11 shows the instantaneous harvested electrical power for $\mathrm{S} 23\left(P_{e}=\eta u \omega\right.$ where $\eta$ is defined in Eqn. 10). As explained in the previous chapter, the coefficient $\eta$ represents the product of the average efficiencies of each PTO stage. The value suggested by Wavestar is $\eta_{0}=0.7$.

Improvements brought by the MPC strategies are particularly easy to notice on Figure 11 corresponding to sea state S23. Indeed, Eqn. 10 with PTO efficiency taken into account via $\eta_{0}=0.7$ implies that the power drawn from the grid costs roughly $50 \%$ more than power fed to the grid. Since MPC is a model-based control, it naturally avoids large reactive power excursions (negative, in the figure), but allows them nonetheless to harvest more power in generator mode. It is interesting to see that the extracted power is a lot larger with MPC than with the reference PI during the transition from S2 to S3, where the PI gains are not optimized anymore for the current sea state.

Computational Cost One of the main concern prior to MPC validation tests was the compliance with real-time imple- 


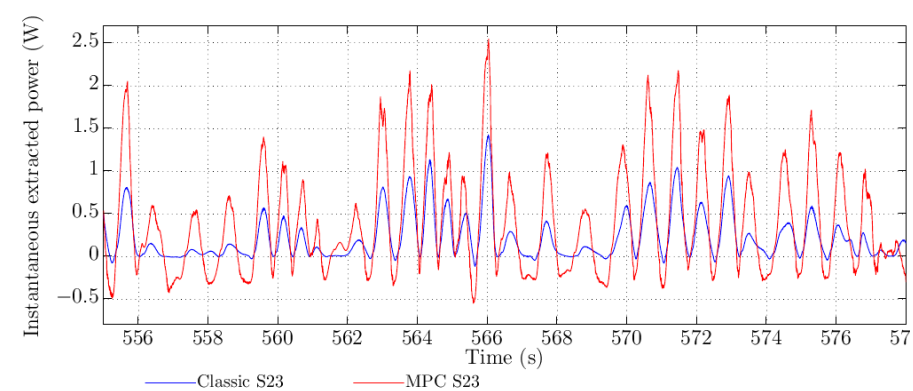

FIGURE 11: HARVESTED POWER FOR S23.

mentation constraints of this kind of control strategy, which has a much higher computational cost than PI control, due to the embedded iterative optimization procedure performed at each sampling time. In principle, MPC could use up to 50ms (its sampling period) to complete its calculation. But this is only true if a specific task were alloted to MPC in a multi-tasking realtime operating system. In practice, the real-time computing constraints proved to be more stringent. To avoid CPU overloads, the Simulink model running on xPCTarget, the real-time operating system used for the tests, had to be completely executed in a time shorter than the base fixed-step size of $1 \mathrm{~ms}$. During the MPC tests, it was observed that the "Task Execution Time" was around $145 \mu \mathrm{s}$, which is still far from the $1 \mathrm{~ms}$ available. Notice that the CPU of the XPCTarget PC used for the tests is relatively slow, as data logging and the other algorithms currently implemented on it do not require a particularly powerful processor.

\section{CONCLUSION AND FUTURE WORK}

In this paper we have presented experimental results obtained on a laboratory device installed in a tank test facility of Aalborg University, which is a scaled-down version of the wellknown Wavestar machine located in the Danish North Sea, near Hanstholm.

The tests were aimed at validating a nonlinear MPC control system for this kind of wave energy converter and assessing its performance with respect to the reference control strategy favored by Wavestar, a proportional-integral velocity feedback control, with gains optimized for a set of sea states.

The full MPC control system that has been tested included an algorithm to estimate the wave excitation torque online from the available sensors and another one to compute short-term predictions of this torque based on time series of past values. The former has proved to yield accurate estimates with no noticeable lag with respect to the wave excitation measurements obtained in dedicated experiments. The latter has proved to be adapted to time-varying sea states and quite effective in the real-time application, though its performance (prediction error) deteriorates towards the end of the prediction horizon.
As to the control algorithm itself, to the best of our knowledge, this the first successful real-time implementation of such a complex, nonlinear MPC strategy, capable of taking into account PTO efficiency, for a WEC. Moreover, it is noteworthy that the small scale of the prototype makes the real-time computing constraints more severe than they would be on a full-scale machine.

Overall, the results show the high potential of improvement brought by MPC in terms of harvested power. The proposed MPC strategy, with different calibrations, outperformed classical PI velocity feedback control optimized offline for each sea state, with gains ranging from $7 \%$ to $81 \%$.

The best results for MPC were obtained when its parameters were retuned to cope with the slow PTO dynamics, neglected in the control model. Of course, for a completely fair comparison, the reference PI controllers could have been optimized accordingly (if time had permitted). Moreover, the most impressive performance gain has been obtained on the time-varying wave S23 in comparison with a fixed-gain reference controller. It could be of interest to make a comparison against an automatically gainscheduled PI controller (which is yet to be designed).

In our opinion, however, the margin of performance improvement is much larger on the MPC side. First of all, MPC parameters were only partially optimized for PTO dynamics, because the (time-consuming) offline optimization had to be terminated before completion, due to the limited time frame of the tests. Moreover, much better results can be expected if PTO dynamics were included in the control model and the MPC design upgraded with it. Better wave excitation torque predictions towards the end of the prediction horizon should also lead to an increase of electricity production.

Further developments and tests, both on the laboratory prototype and in simulation, will certainly be needed to better understand MPC behavior and improve its performance.

\section{REFERENCES}

[1] Chozas, J., 2015. "International LCOE for ocean energy technologies”. Ocean Energy Systems, IEA.

[2] Vidal, E., Hansen, R., and Kramer, M., 2014. "Control performance assessment and design of optimal reactive control to harvest ocean energy". IEEE Journal of Oceanic Engineering, Feb.

[3] Budal, K., and Falnes, J., 1982. "Wave power conversion by point absorbers: a norwegian project". International Journal of Ambient Energy, 3(2), pp. 59-67.

[4] Babarit, A., and Clément, A., 2006. "Optimal latching control of a wave energy device in regular and irregular waves". Applied Ocean Research, 28(2), pp. 77-91.

[5] Babarit, A., Duclos, G., and Clément, A., 2004. "Comparison of latching control strategies for a heaving wave energy device in random sea". Applied Ocean Research, 26(5), pp. 227-238. 
[6] Saupe, F., Creff, Y., Gilloteaux, J.-C., Bozonnet, P., and Tona, P., 2014. "Latching control strategies for a heaving buoy wave energy generator in a random sea”. In World Congress, Vol. 19, pp. 7710-7716.

[7] Hals, J., Falnes, J., and Moan, T., 2011. "Constrained optimal control of a heaving buoy wave-energy converter". Journal of Offshore Mechanics and Arctic Engineering, 133(1), p. 011401.

[8] Cretel, J. A., Lightbody, G., Thomas, G. P., and Lewis, A. W., 2011. "Maximisation of energy capture by a waveenergy point absorber using model predictive control". In Proceedings of the 18th IFAC World Congress, Milano, Italy, Aug, pp. 3714-3721.

[9] Li, G., and Belmont, M. R., 2014. "Model predictive control of sea wave energy converters-part i: A convex approach for the case of a single device". Renewable Energy, 69, pp. 453-463.

[10] Ferri, F., 2014. Wave-to-wire modelling of wave energy converters: Critical assessment, developments and applicability for economical optimisation: Revised version.

[11] Fusco, F., and Ringwood, J. V., 2013. "A simple and effective real-time controller for wave energy converters". Sustainable Energy, IEEE Transactions on, 4(1), pp. 21-30.

[12] Kracht, P., Perez-Becker, S., Richard, J.-B., and Fischer, B. "Performance improvement of a point absorber wave energy converter by application of an observer-based control: Results from wave tank testing".

[13] Ling, B. A., 2015. "Real-time estimation and prediction of wave excitation forces for wave energy control applications".

[14] Fusco, F., and Ringwood, J. V., 2010. "Short-term wave forecasting for real-time control of wave energy converters". Sustainable Energy, IEEE Transactions on, 1(2), pp. 99-106.

[15] Shook, D., Mohtadi, C., and Shah, S., 1991. "Identification for long-range predictive control". Control Theory and Applications, IEE Proceedings D, 138(1), pp. 75-84.

[16] Sanchez, E. V., Hansen, R. H., and Kramer, M. M., 2015. "Control performance assessment and design of optimal control to harvest ocean energy". Oceanic Engineering, IEEE Journal of, 40(1), Jan, pp. 15-26.

[17] Cretel, J., Lewis, A., Lightbody, G., and Thomas, G., 2010. "An application of model predictive control to a wave energy point absorber". In Control Methodologies and Technology for Energy Efficiency, Vol. 1, pp. 267-272.

[18] Hals, J., Falnes, J., and Torgeir, M., 2010. "Constrained optimal control of a heaving buoy wave-energy converter". J. Offshore Mech. Arct. Eng., 133 (1).

[19] Brekken, T., 2011. "On model predictive control for a point absorber wave energy converter". In PowerTech, 2011 IEEE Trondheim, pp. 1-8.

[20] Soltani, M., Sichani, M., and Mirzaei, M., 2014. "Model predictive control of buoy type wave energy converter". In Proceedings of 19th IFAC World Congress, International Federation of Automatic Control (IFAC).

[21] Li, G., and Belmont, M. R., 2014. "Model predictive control of sea wave energy converters part i: A convex approach for the case of a single device". Renewable Energy, 69(0), pp. $453-463$.

[22] Saupe, F., Gilloteaux, J.-C., Bozonnet, P., Creff, Y., and Tona, P., 2014. "Latching control strategies for a heaving buoy wave energy generator in a random sea". In IFAC World Congress 2014, Vol. 19, pp. 7710-7716.

[23] Tona, P., Nguyen, H.-N., Sabiron, G., and Creff, Y., 2015. "An efficiency-aware model predictive control strategy for a heaving buoy wave energy converter". In Proceedings of the European Wave and Tidal Energy Conference (EWTEC) 2015. 